(C) World Scientific Publishing Company \&

Asia-Pacific Society for Computers in Education

\title{
CONVERGENCE OF DATA SOURCES IN THE ANALYSIS OF COMPLEX LEARNING ENVIRONMENTS
}

\author{
SUSANNE P. LAJOIE* and GENEVIEVE GAUTHIER \\ Department of Educational and Counselling Psychology \\ McGill University, 3700 McTavish St. \\ Montreal Quebec Canada H3A1Y2 \\ *susanne.lajoie@mcgill.ca \\ JINGYAN LU \\ Division of Information and Technology Studies \\ Faculty of Education, University of Hong Kong \\ Pokfulam Road, Hong Kong
}

\begin{abstract}
Learning in technology mediated learning environments is a complex process that varies across individual and group contexts. Complex learning environments that are mediated by technology require distinct concurrent methodologies that reveal when and where learning may occur. This paper describes the analysis of two technology-mediated problem-solving environments, one that uses concurrent methods to identify expertise, and the other that examines the influence of technology in a collaborative learning situation. The first example examines individual problem solving in the context of a standalone environment, BioWorld, whereas the second example examines the joint production of medical decisions with traditional and interactive whiteboard technology in a medical classroom. These examples demonstrate how concurrent methods add to our understanding of individual learning as well as the co-construction of knowledge in the context of clinical reasoning using technology.
\end{abstract}

Keywords: Ill-structured problem solving; medicine; collaboration; decision making; expertise; computer-based learning environments.

\section{Introduction}

The National Research Council (2001) describe how advances in the cognitive sciences can inform new approaches to measurement and assessment design. Most importantly they describe how empirical studies of learners have resulted in domainbased models of learning and performance, i.e. in math and science, and these models can guide assessment. We are interested in creating domain-based models of learning in medicine. This paper describes our approach to building models of the individual learner as well as co-learners engaged in medical problem solving using technology. We describe concurrent methods (Creswell, 2003), a mixed method approach consisting of both quantitative and qualitative data, to describe 
and interpret medical problem solving. These data consist of verbal think alouds of individuals and discourse analyses of small groups, computer data collected dynamically during problem solving, and video data of the human-computer interactions. We describe how these multiple sources of data are triangulated to cross validate our interpretations of medical problem solving (O'Donoghue \& Punch, 2003) as a method of providing convergent validity (Campbell \& Fiske, 1959) of the data at hand. Convergent validity refers to the fact that methods that should be related are related and they tell you similar things about the phenomena in question. We describe how data can be combined from different sources to provide a more complete picture of learning and performance in two different medical settings.

It is apparent that a single test score cannot account for complex learning. Indeed multiple forms of evidence are needed to account for the richness and depth of learning in many settings. Convergent methods can be used to provide such evidence. Researchers in the area of assessment have struggled with this issue for some time and several papers address the need for multiple forms of evidence to be used to make better inferences regarding the nature of learning. One example of this is the evidence centered assessment approach that uses complex performance models to make valid inferences regarding learning in context (Mislevy, Steinberg, Breyer, Johnson, \& Almond, 2002). Participant performance models are created, tracked and updated as evidence is collected pertaining to specific proficiencies. These proficiency models are used to guide both instruction and remediation based on such assessments. The complexity of assessment increases with the variety of data gathering techniques and assessment contexts. However, the assessment advantages also increase in terms of providing a better understanding of how complex performance evolves (Mislevy, 2007).

The interdependence of the learning context and assessment has been referred to as a systemic approach to assessment (Frederiksen \& Collins, 1989) whereby learners can use tests or assessments to reflect on their strengths and weaknesses. The transparency of assessment criteria is a first step in helping learners define their goals. In such cases assessments can both promote and describe learning outcomes (Shepard, 2000). Shepard (2000) calls for a new learning culture where assessment is viewed as a reward for what one has demonstrated with regard to learning rather than a punishment for not learning everything. This philosophy of assessment is influenced by Vygotsky (1978) who recognized the difference between what students actually demonstrate on a test versus what their potential for performance was when given assistance. He termed the difference between actual and potential performance the zone of proximal development. The true potential in a problem-solving situation is best assessed in this zone by supporting learners with hints or feedback. Assessment needs to be dynamic or on-going so that assistance is provided in the context of the activity when and where it is needed. The purpose of continuous monitoring is to improve learning during actual problem solving (Pellegrino, Chudowsky, \& Glaser, 2001). Immediate feedback can then be provided to learners when and where they need assistance in the context of the actual target task. Dynamic assessment implies 
that human or computer tutors can evaluate transitions in knowledge representations and performance while learners are in the process of solving problems, rather than after they have completed a problem (Lajoie \& Lesgold, 1992). Scaffolding by teachers, adults, peers or computer tutors eventually needs to fade away gradually so that individuals can demonstrate what they can do as they dynamically gain independent proficiency (Collins, Brown, \& Newman, 1989; Pea, 2004; Woods, Bruner, \& Ross, 1976).

\section{The Nature of Evidence}

When considering diverse indicators of learning and performance multiple mediums of assessment can be an asset. Collins, Hawkins and Frederiksen (1994) went as far as suggesting at least three different mediums of assessment, paper and pencil, video and computer. Paper and pencil tests are associated with traditional forms of assessment of facts and concepts, problem solving ability, text comprehension, etc., but can include less traditional means, i.e., diagrams, graphs, or writing tasks that are required in daily life. Collins et al. (1994) further outline how video can be used to assess student communication, explanation, summarizations, argumentation, listening, question asking and answering skills. Video analyses have the added value of being used after the fact to examine more interactive or social aspects of performance such as joint problem solving and collaborative skills used while working in small groups. Computers provide an alternate form of assessment where student learning can be tracked on specific learning criteria and dynamically assessed in the context of learning as mentioned above (Lajoie \& Lesgold, 1992).

In the next section, we describe how multiple media are used to provide multiple indicators of learning and performance in two medical settings. Our goal is to demonstrate how concurrent methods can be used to analyze multiple forms of data in a manner that converges the evidence into a coherent representation of learning and performance.

\section{Case Based Learning and Teaching in Medicine}

Medicine, as a discipline, has a long-standing tradition of modeling expert diagnostic reasoning and problem solving through tutorials based on real patient cases. A case presentation in medicine generally consists of a detailed analysis of a patient case, but depending on the instructor's prior experience and the facilities in which the patient is seen the solution to these cases varies substantially. Cases are said to facilitate learning for a number of reasons. The first reason is that cases promote problem based learning by providing contexts for applying principles and theories of learning as well as platforms for evaluating possible solutions (Derry \& Hmelo-Silver, 2005). Case based reasoning theory (Kolodner, 1993; Kolodner, Cox, \& GonzalezCalero, 2006; Schank, 1998) describes how cognitive processes are intertwined with memory systems in that prior knowledge is accessed in order to index relevant information and to match new and similar cases to existing memory. Authentic 
contexts are richer and more memorable suggesting that educational situations that embed learning in realistic problem solving experiences will make learning more accessible in the future (Kolodner et al., 2006).

One instructional approach that formalizes such tutorials is the Problem-Based Learning (PBL) approach (Barrows, 1986) where "real-life" cases are presented and students are required to define the problem, create hypotheses, gather and analyze data, and evaluate or justify solutions collaboratively. A major goal of the PBL approach in medicine is to train practitioners to function cooperatively in real-world problem-solving situations during their medical careers.

The examples presented in this paper are anchored in the context of medical case-based teaching where case presentations are used as a form of instruction. In the first example we discuss BioWorld, a computer-based learning environment that presents medical cases in an interactive environment where medical students and physicians can test their diagnostic reasoning skills (Lajoie, 2007, 2009). In the second example, we describe an innovative learning activity called the "deteriorating patient" (DP) designed by a medical instructor (Wiseman \& Snell, 2008) for internal medicine students doing a rotation in emergency medicine.

\section{Solving Patient Cases in a Standalone Computer Based Learning Environment}

BioWorld, is a computer based learning environment that was first designed to promote scientific reasoning in high school students by providing a realistic environment for them to learn about diseases through specific patient cases for them to solve (Lajoie, Lavigne, Guerrera, \& Munsie, 2001). It has been modified for medical populations and we are currently exploring cohort differences (Lajoie, 2003, 2007). In our attempts to create and develop valid cases in medical education for both research and teaching purposes, we have experimented with different methodologies and scenarios to structure case creation as well as validate case solution strategies (Lajoie, Gauthier, \& Richard, 2007). We began by examining medical tutors in their natural setting to see how they instruct students using case presentations and found that they used specific models and scaffolds pertinent to the case in question along with more general types of feedback intended to engage students in self-regulation (Lajoie, Faremo, \& Wiseman, 2001). These scaffolds are faded once students indicate self-sufficiency. Medical tutors use modeling, scaffolding and fading which are key components of a cognitive apprenticeship approach (Collins, Brown, \& Newman, 1989) to help students learn in the context of participating in the practice of medicine.

We chose to extend these results by formally studying medical instructors' instructional discourse and problem solving skills in the context of presenting patient cases using BioWorld. Our analyses of such data will result in expert performance models that can inform the design process so that appropriate student assessment and feedback can be provided to learners. Identification of cognitive competencies 
is the first step in developing better instruction, given that tutors, human or computer, could be alerted to where and when more scaffolding and/or feedback may be needed in the context of a clinical case.

In this interactive learning environment participants formulate diagnoses about patient cases as they order diagnostic tests, request information about the patient vitals, visit an on-line library or ask for assistance. For this particular study, we were interested in how medical pedagogical experts solve a set of BioWorld cases and explain their thinking during the problem solving process as they would to their students as they solve cases. We were interested in two things - validating individual reasoning traces, and examining how experts, as a group, converged or diverged in their solution traces. The usefulness of the former is to ensure that researchers have interpreted expert reasoning appropriately, whereas the advantage of the latter is to establish expert consensus on a case-by-case basis. Visual representations of the problem solving space were created for each expert on each case and included plans, actions and paths taken to reach a diagnosis. In addition to using such representations to describe problem-solving activities we used these representations retrospectively with participants as a method of validating our interpretation of diagnostic reasoning. Participants could review the visual summary of their problem solving process, and add or delete items from their solution representations. As with most ill-structured problem solving activities there is more than one way to reach an adequate solution. The analyses were designed to provide an accurate representation of how experts diagnosed patient cases. Analyzing multiple experts' performance for each case helps establish a more complete problem space of competent performance, where solution sequences were identified and compared in terms of convergence or divergence in expert actions and explanations. This analysis would serve to build a more complete model of expertise underlying each case where the diversity among expert problem solvers could be represented as opposed to a single and potentially underrepresented expert solution path. We describe the methodology below.

\subsection{Methodology}

Structured think-alouds were conducted with five medical expert teachers on three patient cases as they used BioWorld. Medical instructors routinely and naturally "think aloud" as they present patient cases to students. Thus, these individuals are ideal candidates for articulating their knowledge and thought processes while solving cases. We asked them to solve and teach cases using BioWorld. The data collection process involved two separate meetings, with each expert. In the first meeting, participants solved three cases and various data sources were collected for each case. Verbal think alouds were recorded through the use of a screen capture program and computer actions were recorded by computer logs. This combination of digitized data along with the researcher's observations helped contextualize participant actions as they interacted with each case moment-by-moment. These multiple data 
sources provided complimentary types of evidence about how the expert reasoned about the patient case. Our challenge was to integrate the data into a coherent and accurate visual representation of the participant's problem space. Our research goal was to use multiple data sources to create reliable and valid interpretations of the diagnostic reasoning processes produced by the experts. The researcher first transcribes the verbal data into transcripts and then combines the verbal transcript and computer log into one protocol.

As shown in Figure 1, the individual's computer log is inserted into the verbal transcript and appears in bold within brackets, for example on line 11 "[few months add evidence]" is added after the transcript lines 7-10 that state "and her problem may or may not be related to ah pregnancy... well for a few months which suggests that her problem is not acute."

After adding evidence in line 11 the participant states the "problem started when she began taking medication, ah which suggests the possibility that some of the ah unwellness relates to an adverse affect of the medication." The researcher now has the complete context for the reasoning and can review this merged data, knowing what the participant said as well as the actions that they took in Bioworld while reasoning. The researcher uses this protocol to construct a summary of video clips that are later used to build an associated visual representation of the problem state during the solution process. The protocol is first segmented into small meaningful idea units. These lines of transcript are used to justify specific nodes in the visual representation. Consequently, every node in the multi-layered visual representation is justified by a series of original statements coming from the transcript.

Transcription and coding were done using Transana software (Woods \& Fassnacht, 2007). Transana supports the ability to attach time markers to both audio and video segments, providing a mechanism to coordinate information sources and a way to refer back to segment units where you can hear or watch participants as they interacted with specific features of Bioworld. Cmap (Novak \& Cañas, 2006), is the concept map tool that we used to construct the multi layered visual representation of the case resolution. Utilizing a visual representation to summarize data for analysis purposes is not new (Henderson, Yerushalmi, Heller, Heller, \& Kuo, 2003). However, in our context multiple data sources were analyzed to create a representation that participants could use to reflect on their own medical reasoning. Participants could interact with their own decision paths, categorizing their paths in terms of what was most essential to the diagnostic process. As part of our analyses we chose to merge the expert representations to provide evidence of commonalities and differences in their reasoning paths.

The first phase of the analysis was data integration from the multiple methods used in this study, video data, verbal transcripts, and computer trace data. The data integration was used to create a visual representation of the expert solution process. Each representation provided contextual information as to what experts said in the context of using BioWorld to collect data and make decisions. Consequently, the representation served to externalize the expert's reasoning processes so they could 


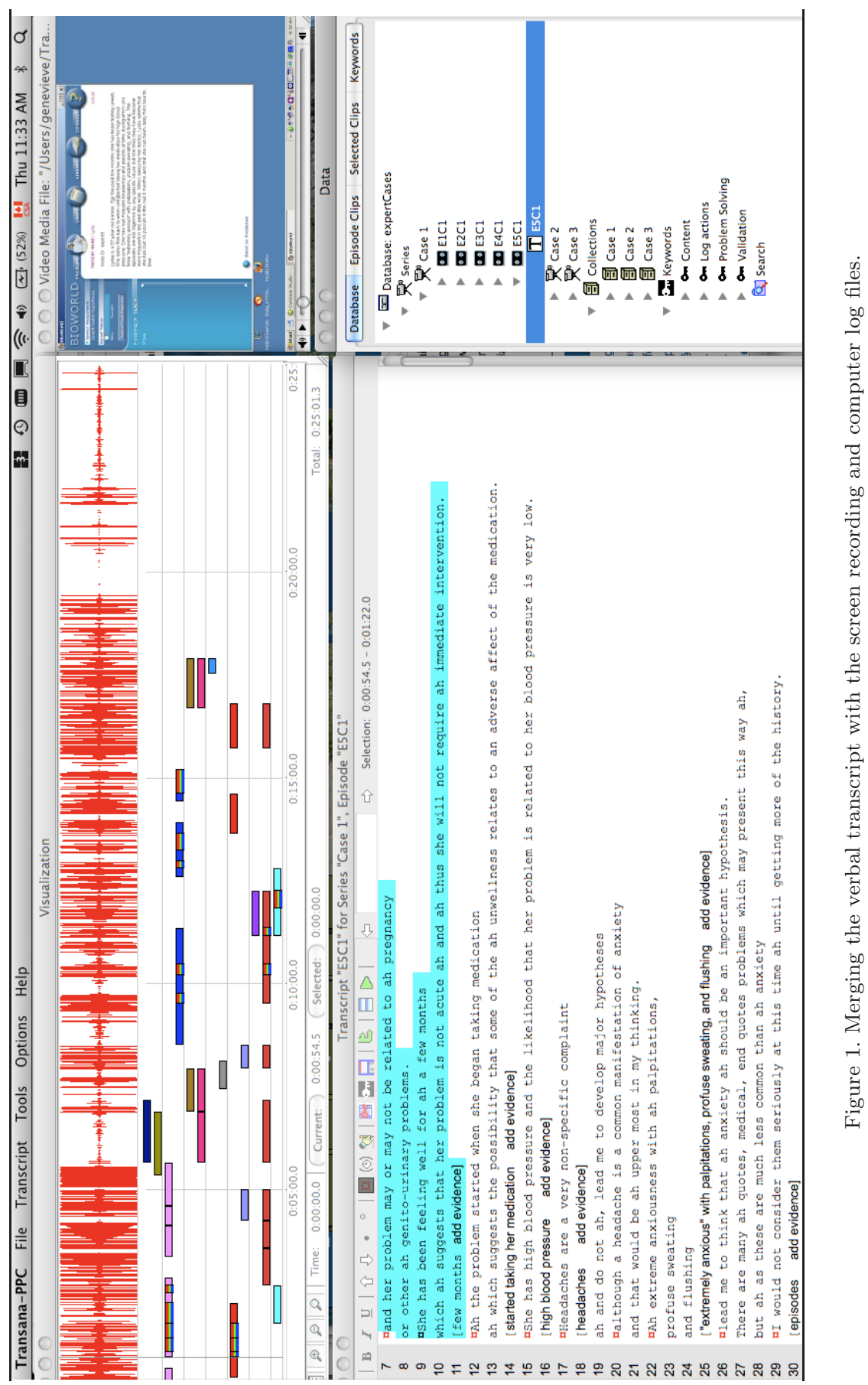


reflect on their own diagnostic reasoning. As a way of validating the researcher's interpretation of the data, participants were asked to inspect their own representations and verify the content, modifying or adding information that might have been missing. The participant interpretation would supercede that of the researcher. The visual representation is a sequential summary of the researcher's transcript of their problem solving. Participants could see their original transcripts by mouse-overs as shown in the screen capture of Figure 2. The square box reveals the transcript associated to that node.

Participants were then asked to categorize elements of the reasoning process that were absolutely necessary (crosshatched background), the ones that were necessary (lines background), and the one that were useful (dotted background) to solve the case. We collected data on their validation of the summary, their changes and their classification of elements in the representation as well as observation notes.

Participants took an average of 15 minutes to complete their validation of each case. All participants were surprised about some of their own actions or explanations. They all used the verbal transcript inside the nodes to verify what they had said and to refresh their memories. After validating the summary, participants made

Problem solving path of Expert 4: Lydia - Log Data/Verbal Protocol - V2

39 yr old banker

past few months she has been feeling unwell

taking medication for high blood pressure
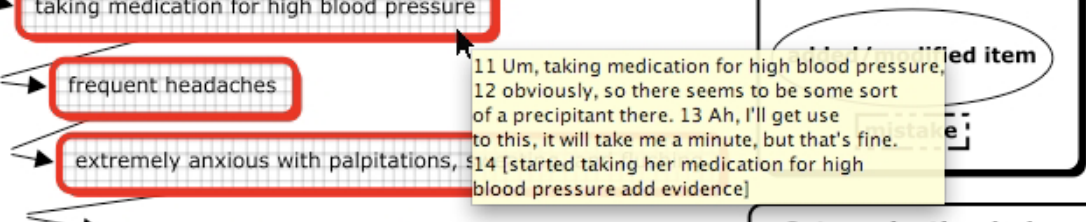

symptoms more frequent:

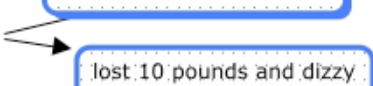

Categorization index

lost 10 pounds and dizzy

select Hypothesis Rheochromocytoma

Validation index

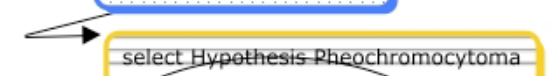

absolutely necessary

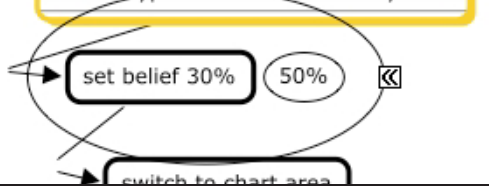

nescessary information

useful information

Figure 2. Extract of visual representation of Expert 4 path with mouse-over. 
very few changes regarding the accuracy of the summary, confirming that researcher interpretations and representations were reasonable. More specifically for patient case 1 "Lydia" which had a diagnosis of pheochromocytoma, 3 of the 5 participants made changes ( 2 to 5 in total) to the visual representation. It is noteworthy that there were very few changes in the validation phase, indicating that the researcher was able to converge these multiple forms of evidence (verbal think alouds, computer log files, and screen recordings) into a coherent and accurate visual representation of the diagnostic reasoning process. However, changes that were made consisted of modifications regarding beliefs about diagnoses, adding relevant diagnostic tests, etc. Figure 2 presents a portion of the changes and categorizations made by Expert 4 for case 1 . In this situation, the expert modified his original confidence about his diagnosis from $30 \%$ to $50 \%$ confident. He also categorized three pieces of information as absolutely necessary in determining the diagnosis: taking medication for high blood pressure, frequent headaches, and anxiety with palpitations, sweating and flushing.

Expert consensus was explored to determine the variability between expert decisions and actions taken to reach a diagnosis on a patient with pheochromocytoma. Data from 5 experts are provided in Figure 3 that illustrates each expert's sequence along with groupings of elements that experts had in common. This combined problem space graph demonstrates where participant actions and reasoning converge and where they diverge. One potential use of such representations might be to study their usefulness as learning resources for instructing more novice problem-solvers in complex problem solving situations. A more obvious one is simply to help us better understand expert problem solving in such domains.

The sequential representation allows us to browse and compare the similarities and differences at the macro level of the decision process without excluding micro level differences. At the micro level, participants reasoning and verbal explanation are nested. We can appreciate that all five experts identify and talk about the same three vital signs, yet they talk about it in different ways. The way they link elements together and create evidence constellations provides more information than processes in isolation. This is especially true for sets of symptoms or sets of diagnostic tests results. Overall when we compare and contrast experts there is consistency in the type and quantity of evidence collected, the list of hypotheses generated (verbally and with computer logs) and the time required for the formulation of the correct hypothesis. However, we found differences in the number and list of tests ordered, as well as the length and level of explanations. The use of the visual representation helps us better understand where in the sequence of events each expert differs. For example, E2 was the first to suggest a hypothesis. Other experts tended to collect more evidence prior to considering a diagnosis. The information on the possible differential diagnoses (multiple competing hypotheses) is important for instructional purposes since feedback can be provided about such diagnoses in the context of their solution traces. 


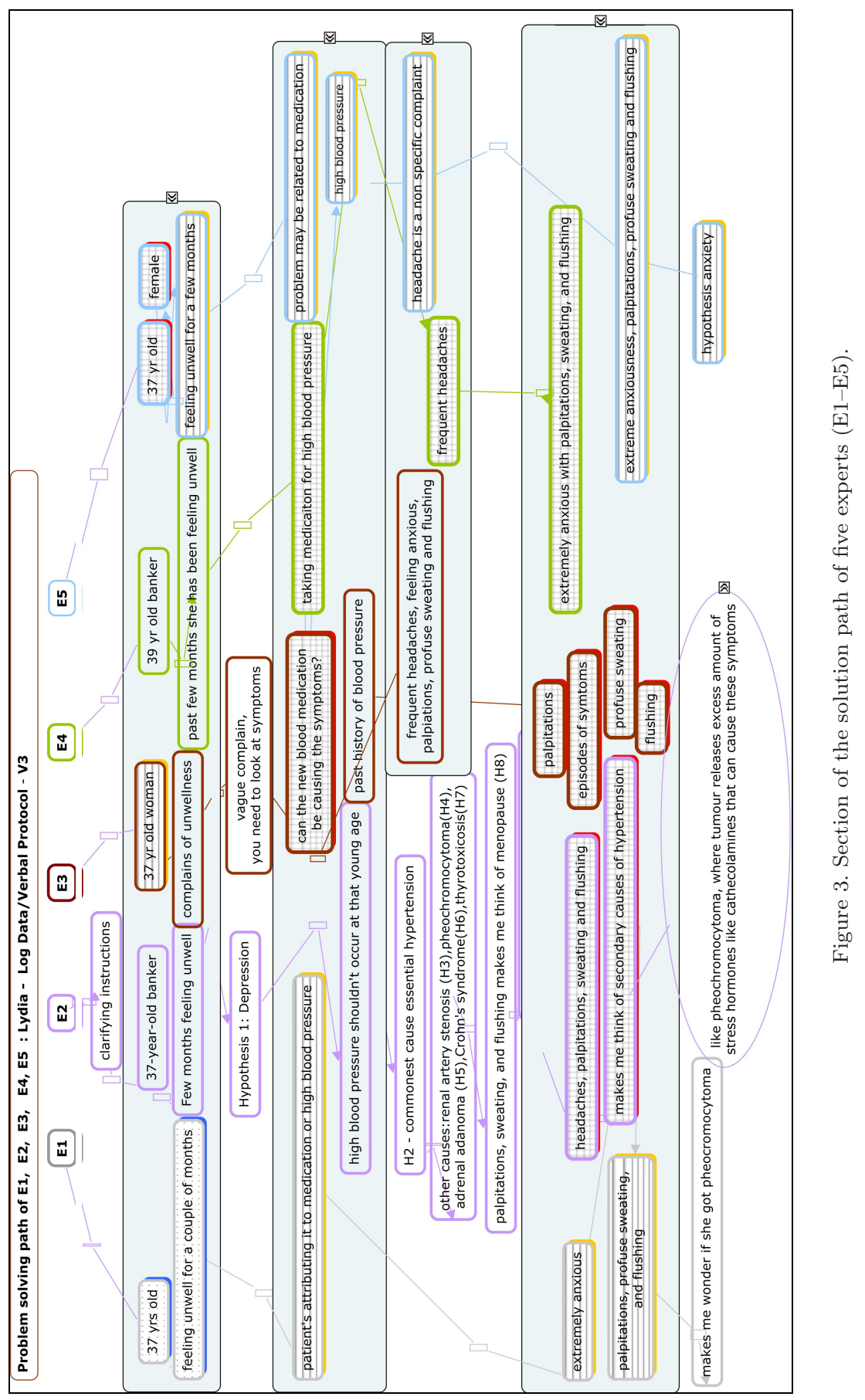




\subsection{Analytical challenges and outcomes: Knowledge and performance elicitation from experts}

Creating a model of proficient performance in the context of solving a case in an open ended learning environment presents numerous challenges. The "value added" of the approach taken in this study is that the multiple forms of data helped provide a more complete picture of the diagnostic reasoning process. Computer trace data alone forces researchers to make some inferences about the reasoning process, in terms of the underlying reasoning behind plans and actions. The addition of the think aloud data provided us with a broader lens to interpret expert reasoning. By framing the problem solving task as a case presentation activity the medical instructors perceived the task as authentic and made the think aloud process more natural.

Our next step is to use these expert models in a systematic manner with medical students. We have started to use the visual representations as a form of feedback for novice learners where they review them after they attempt a case and reflect on how their solutions may have differed from an expert's. Medical students can read and listen to what experts were thinking in the context of diagnosing a patient case. We are interested in developing a more fine-grained analysis of learner differences along the proficiency dimension. We will do this by examining different cohorts of medical personnel using think-aloud protocols to examine the breadth and depth of their problem solving abilities. The analyses of such data will result in a fine tuned model of where computer-tutoring feedback should be considered in the context of specific patient cases. These types of analyses will lead to more refined types of assessment that can be used more dynamically in computer based learning environments.

Up to this point in the paper we have emphasized concurrent methods that can be used to document the individual problem solving experience in the context of a stand-alone problem-solving environment. We have also demonstrated how these methods can lead to an interpretation of both the individual and a group of experts who solve the same case. Our goal has been to describe the complexities of documenting an expert's diagnostic reasoning processes by using multiple methods of assessment. In the next section we discuss the challenge of studying interactive group learning situations in the context of a medical simulation called the deteriorating patient activity.

\section{Understanding Medical Decision Making in Simulated Settings: Diverse Perspectives and Multiple Methods}

This section reviews a case study of an innovative classroom learning activity used to prepare third year medical students for their rotation in internal medicine. In the activity, the teacher implements role-play scenarios of actual medical emergencies. Students play the roles of various medical residents who must treat a patient whose vital signs have suddenly and rapidly begun deteriorating. The students' job is to stabilize the patient by stopping the deterioration of the vital signs and returning 
them to normal values by discovering the immediate cause of the trouble. If they do the right thing the patient will recover; if they do nothing or they do the wrong thing the patient will become sicker. As the role-play progresses the patient will decline more quickly if students fail to solve the problem. The teacher succeeds in creating classroom learning environments that his students find medically authentic, compelling and pedagogically effective.

The deteriorating patient activity simulates actual medical emergencies that internal medicine students encounter in emergency rooms (Wiseman \& Snell, 2008). The teacher draws on his clinical and pedagogical expertise and acts as a coach as he plays the roles of the deteriorating patient and the duty nurse while challenging and scaffolding his students as they struggle to save the life of the patient. In this simulation the instructor acts as the patient and the nurse and the students take turns as the physician who manages the patient. If the students make incorrect decisions the patient's condition deteriorates. When the students need help they ask other students to step in to solve the problem. The instructor scaffolds the students in the context of their decision-making. Students learn to ask the right questions, order the correct tests, and manage the situation. Students also learn to manage stress and make decisions quickly based on the patient's rapidly deteriorating medical condition, which are key ingredients for success in managing emergency situations.

We introduced collaboration as well as technological support to this simulation with the goal of supporting learning throughout the role-play activity. Several learning theories are exemplified and interrelated in the design of this study. Collaborative learning provides students opportunities for developing social and communication skills and promote deeper level of thinking and shared understanding (Johnson \& Johnson, 1999). Situated learning theory argues that learning best occurs in authentic learning contexts where knowledge is dynamically and collaboratively constructed (Lave, 1991). Situated cognition takes place within a nexus of activities, tools, and culture (Brown, Collins, \& Duguid, 1989). Similarly, constructivist learning theories speak to the interrelationship between private thought and social experiences (Greeno, 1989). In this particular study we investigate whether or not technological support of communication and argumentation during group problem solving leads to better decision-making.

Role-play scenarios posed unique research challenges in that different kinds of data were needed to adequately investigate the role of the teacher, the learners and technology. Concurrent methods were used, providing both qualitative and quantitative data. Videotapes of simulations were transcribed, coded and analyzed with respect to verbal and non-verbal interactions. Computer logs were collected and written annotations were analyzed and coded. Our analyses integrate these multiple sources of data to account for medical problem solving and communication among peers as well as between students and the teacher. The data were analyzed from different perspectives. One perspective examined whether collaboration among peers led to better decision-making. Another perspective examined the role that teacher scaffolding played in the decision making process. Finally, the third perspective examined the relationship between what the teacher said, what students said and 
wrote, and the role of technology in these processes. Given that these authentic learning activities are socially situated, they cannot be examined in isolation from communicative interactions or from the influence of technology tools in supporting these interactions. We describe how these multiple sources of data converge and we examine this convergence from multiple analytical frameworks.

\subsection{Methodology}

Fourteen third year medical students were recruited from an internal medicine rotation. The teacher organized students into two conditions, a traditional whiteboard condition (TW) that had no digital technology support, and an interactive whiteboard condition (IW), with technological support that met on separate days. Each condition had three subgroups. The TW condition worked in subgroups where they discussed the case and interacted in the role-play with the teacher one group at a time, while the other groups observed the role play and discussed the case amongst themselves as to what they might do differently. In the TW condition the teacher documented the class contributions to the medical argument on a traditional (frontof-the-class) whiteboard as each group made contributions to the process of how to solve the medical emergency based on student contributions. The IW condition used EBEAM software to interface information between the traditional whiteboard and individual laptops individuals used in their subgroups. In other words, whatever appeared on the original whiteboard also appeared on their laptops. The IW provides a form of groupware that enables groups of participants to collaborate for a common purpose (Khoshafian \& Buckiewicz, 1995). Groupware applications make such collaborations or communications visible. The difference between the two conditions was that in the IW condition the students could annotate the medical argument on their laptops, adding and sharing information they thought relevant to solving the medical problem. Both conditions were asked to solve a learning activity called the "deteriorating patient" (Wiseman \& Snell, 2008).

The teacher as coach posted and updated information about the patient's medical history, medications, and fluctuating vital signs on a whiteboard and also acted out roles of patient and nurse. The whiteboard in both conditions was used to document the patient problem list, which included information about the diagnosis (Dx), patient management, patient vital signs (Pulse, Blood Pressure, Respiration, Oxygen saturation and temperature) and prescriptions (Rx). However, the IW groups could annotate their copy of the whiteboard on their own laptops, providing their comments and suggestions on the existing medical argument. In the TW condition one subgroup is solving the case and being tutored by the instructor, while the other two subgroups are discussing their own medical arguments. In the IW groups, one subgroup is working with the instructor while the other two subgroups are sharing and documenting their arguments via networked laptops posting their arguments on the IW. In the IW condition a dynamic trace of the medical argument is being created cumulatively as both the tutored and observing groups formulate their solutions on the shared whiteboard. The IW condition provides more than a visual 
trace of a medical argument; it provides a cumulative trace of more than one group's reflections on the argument thereby affording additional and augmented opportunities for perspective taking and self-assessment. In the BioWorld work described earlier, we demonstrated the power of identifying and providing expert pedagogical models for student reflection and assessment. In the current study we demonstrate the advantage of peer reflection and shared knowledge that is constructed through consensus building as peers reflect on the problem list as it is documented and scaffolded by the instructor.

Data included protocols of student-student and teacher-student verbal interactions and computer annotations. Protocols of student-student verbal interactions provided evidence of decision-making and communication patterns in the context of argumentation. Protocols of teacher-student verbal interactions provided evidence of the cognitive, social and affective aspects of strategies for scaffolding student problem solving. Computer annotations exposed the IW students' argument building while the other subgroups interacted with the teacher.

\subsection{Data and analysis}

Three research questions were formulated, (a) did technology mediated annotations promote group decision-making and communication and if so, how; (b) did the teacher vary his scaffolding strategies based on the introduction of technology and if so, how; and (c) how is students' decision-making linked to scaffolding and technology usage?

Verbal discourse and computer annotations were analyzed with respect to how the technology mediated the students' communication and problem-solving activities. The challenge in this study was to understand the deteriorating patient context in order to arrive at a meaningful analysis and to interpret the data with respect to how the technology mediated the groups' knowledge construction and decisionmaking.

Qualitative methods were used to analyze verbal protocols and annotations because these protocols contained discourse dependencies that violate the assumption of independence made by most inferential statistics, thereby rendering quantitative methods largely ineffective. Discourse analysis was used to assign verbal protocols and written annotations to four coding categories: decision-making, communication, scaffolding or annotation. Stretches of decision-making discourse were further coded as planning, data collection and managing the patient or interpreting the situation. Stretches of communicative discourse were coded as informative, argumentative, elicitative, responsive, directive or off-task (Saab, van Joolingen, \& van Hout-Wolters, 2005; van Boxtel, 2000). Scaffolding discourse was coded in accordance with its role and function in role-play scenarios. For instance, the teacher as deteriorating patient or nurse helped students to (a) understand and implement the emergency algorithm appropriately, (b) manage patients under emergencies (c) understand hospital hierarchy, (d) develop medical situation awareness and (e) work 
Table 1. Coding schema and definitions of DP activity.

\begin{tabular}{|c|c|}
\hline Category & Definition \\
\hline \multicolumn{2}{|l|}{$\overline{\text { Decision Making }}$} \\
\hline 1. Planning & $\begin{array}{l}\text { Students formulate plans for collecting patient data and for } \\
\text { managing patient condition }\end{array}$ \\
\hline 2. Data collection & Student collect patient data, i.e., physical condition, lab tests \\
\hline 3. Managing patient & Students manage patient's condition \\
\hline 4. Interpreting & $\begin{array}{l}\text { Students interpret patient's condition based on collected data } \\
\text { and management interventions to construct shared } \\
\text { understanding }\end{array}$ \\
\hline \multicolumn{2}{|l|}{ Communication } \\
\hline 1. Informative & Speaker provides information \\
\hline 2. Argumentative & $\begin{array}{l}\text { Utterances may contain the following words like "because", "if", } \\
\text { etc. to indicate reason, condition, consequence, continuation, etc. }\end{array}$ \\
\hline 3. Elicitative & Speaker asks for addressee's opinion \\
\hline 4. Responsive & Speaker reacts to an earlier utterance \\
\hline 5. Directive & Speaker gives an instruction or makes a suggestion \\
\hline 6. Off task & Utterance irrelevant to problem solving task \\
\hline \multicolumn{2}{|l|}{ Scaffolding } \\
\hline 1. Domain knowledge & Providing cognitive guidance to help students solve problems \\
\hline 2. Rule & $\begin{array}{l}\text { Providing social, and affective scaffolding to help students achieve } \\
\text { multiple educational and performance goals }\end{array}$ \\
\hline \multicolumn{2}{|r|}{ 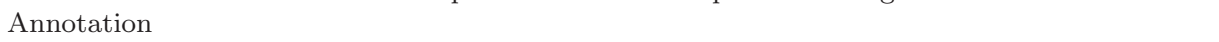 } \\
\hline 1. Proposal & Suggestion for alternative management or test \\
\hline 2. Disagreement & Disagree with decision-makers' management or interpretation \\
\hline 3. Interpretation & Demonstrating interpretation to the patient's situation \\
\hline
\end{tabular}

collaboratively with peers. Annotations were coded as proposals, disagreements or interpretations (see Table 1 for detailed definitions).

\subsection{Results}

The first research question addressed whether groups with technology (IW) differed from groups without digital technology in their decision-making and communication. Differences were found in the type of decisions groups made about patient management and their interpretation of the patient situation. In the early stages of the simulation the IW group put more effort than the TW group into interpreting the situation and less into patient management. In the later stage, as the patient's situation becomes more urgent, the IW group put more effort into managing and less time interpreting the patient situation (Figure 4). For communicative activities, all groups produced more utterances in the early stage, except that the TW group produced more Informative remarks in the Late stage.

The second research question examined the role of human scaffolding in both conditions with respect to when and why the teacher spoke as instructor or in his role as nurse. The teacher spoke as instructor in order to manage group dynamics and to provide domain knowledge and as nurse to give the test results and patient vitals. The nature of the deteriorating patient simulation is that the patient deteriorates 


\section{Decision making activities}

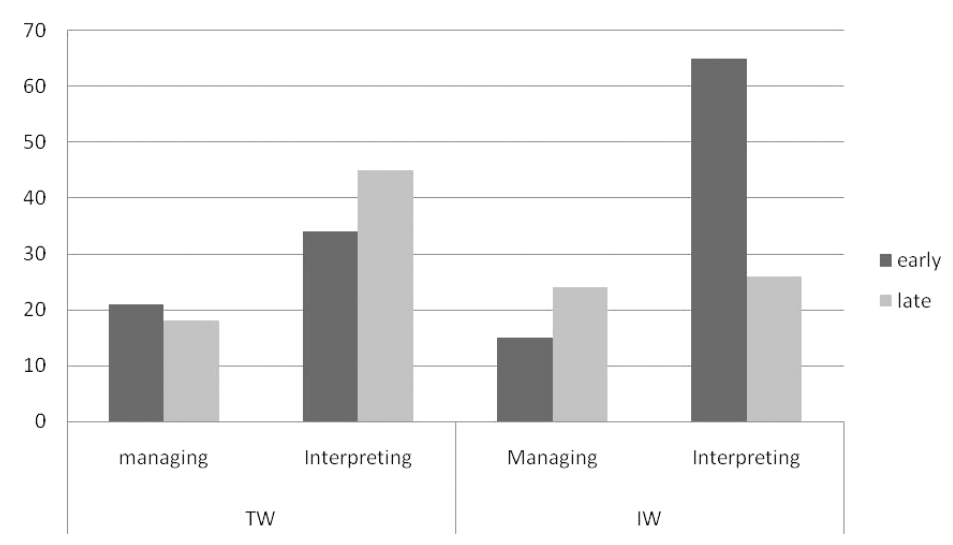

Figure 4. Frequency distribution of decision making activities of TW and IW across early and late stages.

if actions to solve the problem are not taken. Consequently, scaffolding prompts, by instructor and nurse, are most evident in the later stage of problem solving as the patient deteriorates (see Figure 5(a)). However, the content of the scaffolding differed for the two conditions and the quantity varied as well. In general the IW group needed less scaffolding than those who had no technology support. Furthermore, the teacher increased his scaffolding for the TW groups from the early to late stages, with particular help with rules and domain knowledge whereas he decreased such scaffolding for the IW group from early to late stage (see Figure 5(b)).

The final research question examines the connection between decision-making, scaffolding, and the role of technology. One role of technology in this study was to provide a cognitive tool that could be used to build and externalize a medical problem list based on a series of pull down menus. These menus as seen in Figure 6 were used to annotate what was already on the whiteboard. Basically students could annotate the medical argument through these menus or by typing into their laptops. These menus could be seen as guided representations, but the use of the pull down menus was infrequent in comparison to the student generated annotations seen in the Figure 7 . These arguments were shared with other groups through laptops. We examined the relationship between the students' oral discourse and the written shared annotations, and found that oral communicative activities were facilitated by the shared annotations and led to productive decision-making. The findings suggest that interactive whiteboards enabled students to share data and to construct a shared understanding about the patient that led to other communicative acts. Shared visualizations can be used to support verbal interactions, promote productive argumentation and facilitate negotiation that lead to shared understanding. 


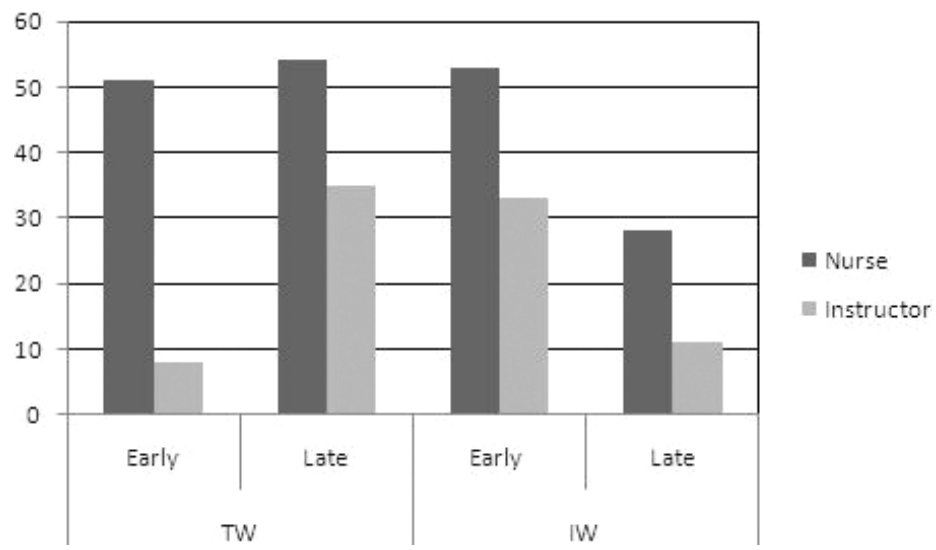

(a)

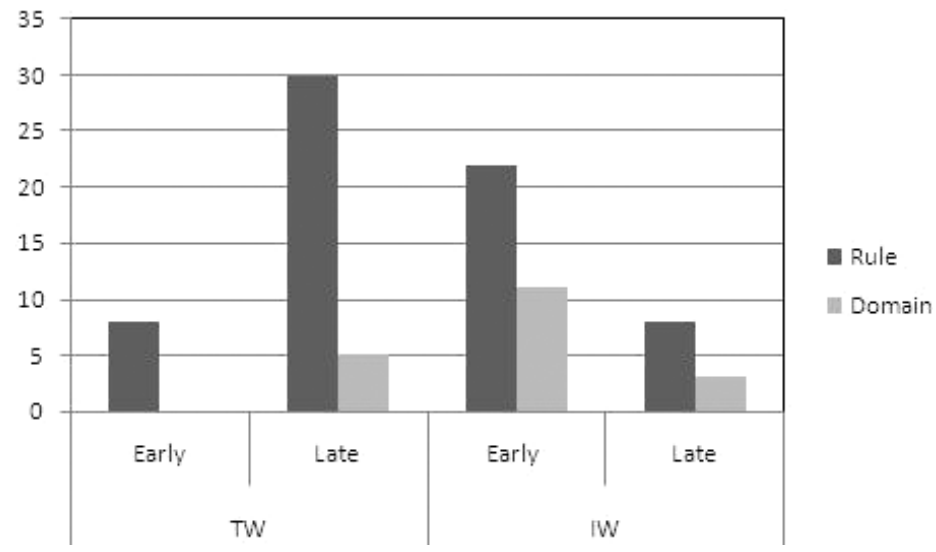

(b)

Figure 5. Discourse patterns by the teacher playing different roles (5a) and using different scaffolding strategies (5b).

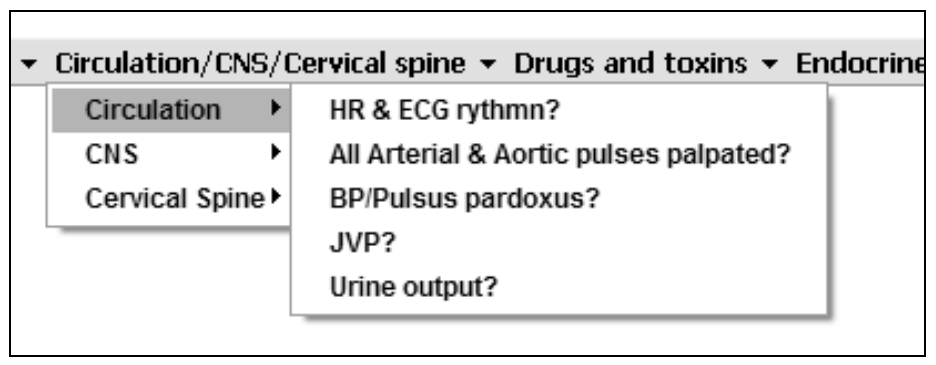

Figure 6. Screen shot of dropdown menu. 
When teams work together in medical emergencies they need to share their understanding of the patient situation in order to manage the patient. Shared understanding (Saab, van Joolingen, \& van Hout-Wolters, 2005) has been defined as: (a) constructing shared understandings of problems, (b) reaching the same hypotheses, (c) sharing the same techniques, and (d) establishing the same conclusions. Several aspects of shared understandings are relevant to the DP study.

Qualitative analysis revealed that students in the IW groups communicated a lot in the early stages of problem solving in order to establish a shared understanding of the patient's situation in order to plan further procedures. In the late stage of problem solving, these students sought to establish shared understanding so as to reach agreements on how to stabilize and manage the patient. The IW visualization and argumentation tools helped students established this shared understanding early on so that less communication was needed later for interpretation, and more time was released to conduct patient management actions. Here is an example of how the IW facilitated decision-making by supporting the development of a shared understanding.

N: I am not sure we should um, give epinephrine.

...

$N$ : He's not responding to fluids.

$Y$ : We should wait for the X-ray first. [As to] epinephrine or vasopressin. I don't know.

N: No. No. You can't wait for the X-ray like when the blood pressure is going down like that.

N: (To T) We're thinking of give like vasopressin or epinephrine.

T: $U m-H m$.

N: Because like waiting for the X-ray and stuff is taking a while and the patient is crashing, so we can bolus. Like is it one milligram epi? One?

T: So you're thinking of giving vasopressin?

N: Yeah.

$Y$ : Or epinephrine.

N: Yeah.

In this discourse "epinephrine", "fluids", and "X-ray" were introduced as annotations by observing IW students who were commenting on actions that were mentioned previously (see Figure 7). By reading the discourse and examining the visual annotations on the screenshot, one can see the direct connection between what was said and what was added as annotations that were shared using groupware. Students were building on other students' arguments demonstrating a shared understanding of what had occurred at a particular point in time. Student N expressed confusion as to whether or not they should "give epinephrine", and responded to an annotation about "bolus" stating that it could not work because "He's not responding to fluids". Student Y suggested they wait for results from the X-ray and was uncertain with respect to "epinephrine or vasopressin" as well. Student N disagreed about "X-ray" 


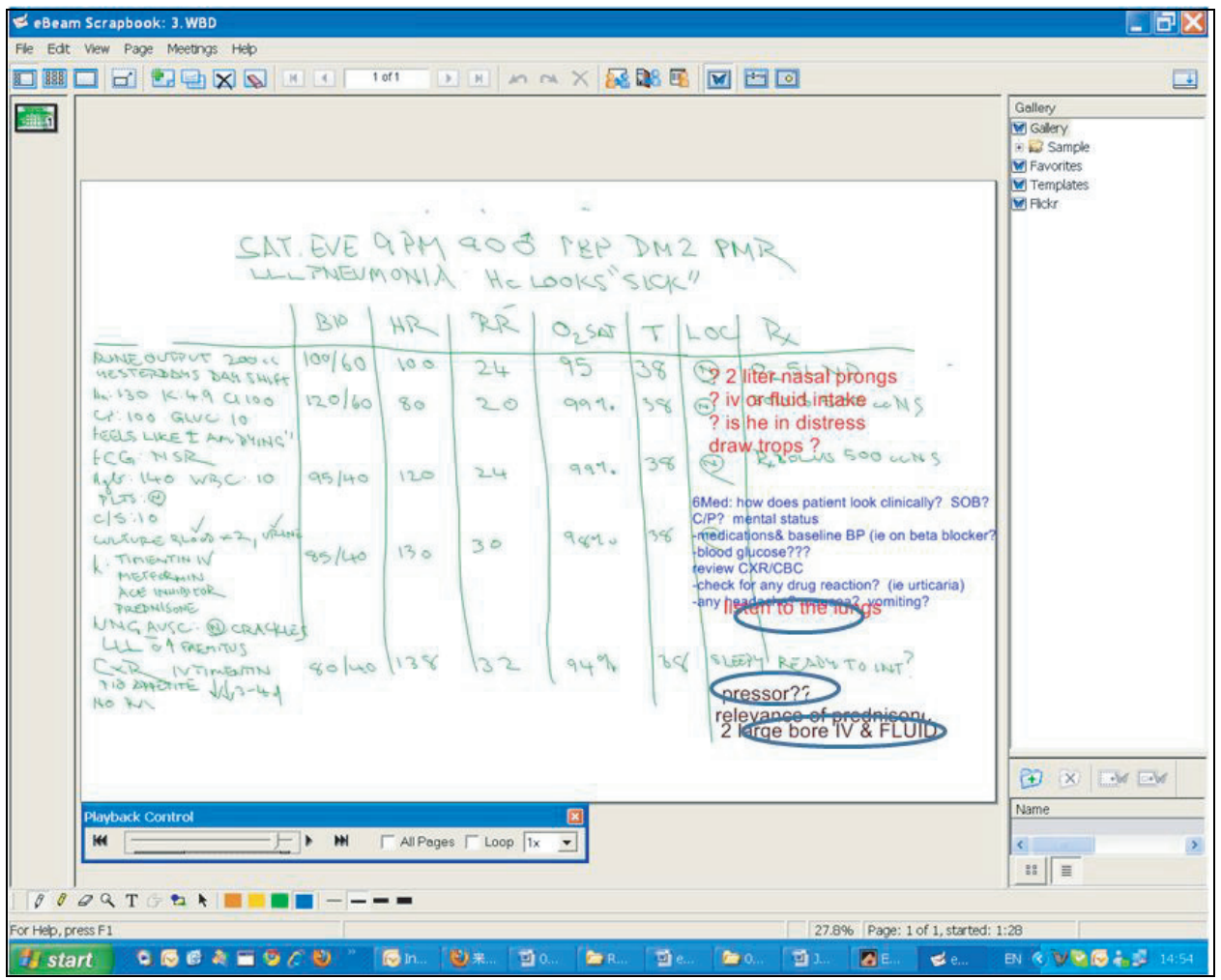

Figure 7. Screenshot of Interactive Whiteboard where students build, annotate and share medical arguments.

and then told the teacher about their plan of giving Vasopressin or Epinephrine. Student $\mathrm{N}$ then explained why she did not agree to "waiting for X-ray". Student Y conceded, added "or epinephrine" and student N agreed. A shared understanding was reached.

Discourse topics leading to the development of shared understandings were often triggered by alternative opinions provided by IW students in the form of shared annotations. Annotations facilitated the construction of shared understandings of the patient and helped students focus on patient management. TW students in the later stage of the DP activity had difficulty developing effective patient management actions and continued to invest significant time and effort in communicating so as to develop shared understandings of the patient's problem. The TW students seemed stuck at the level of trying to simply establish basic shared understandings (Saab et al., 2005).

As stated earlier, the IW group spent more time managing the patient than the TW group, and consequently was more adaptive in a medical emergency. Shared cognition facilitates the construction of shared situation models and joint problem 
spaces that lead to better decision making and problem solving. The introduction of the interactive whiteboard also mediated the teacher's scaffolding strategy. First, the teacher spent less time scaffolding the IW groups then the TW. However, he was also able to build on the shared annotations of the IW groups to broaden his scaffolding to the whole classroom on key elements that were raised in the annotations.

M: We'd like to know what meds he's on.

T: Ok. So meds that he's on. He is on Timentin IV.

M: He is on Timentin.

T: He is on Metformin. He is taking an ACE inhibitor. And he's on Prednisone.

$\mathrm{M}$ as a student asked for medication suggested by the annotation. This question leads the student to pursue questions on possible medication problems. However, instead of giving students the right medication that would have solved the problem, the nurse provided different medications to complicate the situation. Introducing a complication can be a form of problematizing (Reiser, 2004) which is a type of scaffolding that helps students focus on important learning goals. Reiser (2004) suggests that problematizing can enhance the problem solving experience by focusing attention and decision making on issues that may otherwise be overlooked. Problematizing can increase the difficulty of problem scenarios by intensifying the challenges of tasks (Reiser, 2004). In the deteriorating patient activity, the nurse/instructor provides such scaffolding, by complicating the patient's vital signs to draw the student's attention to the more complex problems that may add to problem difficulty but ultimately lead more productive learning. Scaffolding differed by condition. The instructor tended to provide more problematizing in the IW condition and more clarification and verification questions in the TW condition. These findings imply that the teacher's scaffolding becomes more complex as learners become more sophisticated (see also Lu \& Lajoie, 2008).

\subsection{Summary}

The concurrent methods used in this study included: verbal data between students working together in subgroups; student and teacher discourse in the context of the deteriorating patient activity; and computer annotations shared in the IW condition. Together these multiple methods were used to provide evidence of group decision-making, teacher scaffolding and technology tool use in the context of the deteriorating patient activity. If one were just to look at the student-student discourse, one would not see the influence of the teacher's scaffolding. If one were just looking at scaffolding one would not see how the students influenced each other. The addition of analyzing the computer annotations in the context of the discourse demonstrated when students chose to make new plans, collect new data, manage the patient in a particular way or interpret patient symptoms differently. These multiple methods add to our understanding of student-teacher-technology interactions in this complex medical problem solving situation. 
Teaching clinical reasoning is difficult at best, but the deteriorating patient activity did provide a sense of realism that afforded the type of naturalistic decisionmaking required in ill-structured problems. By playing different roles, the teacher was able to scaffold both the cognitive aspects of student argumentation as well as the social and affective elements required for effective group work (Lu, Lajoie, \& Wiseman, submitted). Consequently, it was just as important to analyze the nature of the teacher's communication and how it was used to scaffold learning. We found that students in the technology condition communicated more and established a shared understanding of the task earlier in the activity than the traditional whiteboard condition, and that this communication led to more effective problem-solving (Lu \& Lajoie, 2008). Teacher scaffolding was investigated in both conditions. The teacher scaffolded less in the technology condition and his assistance faded over time whereas the opposite results occurred in the TW condition (Lu \& Lajoie, \& Wiseman, submitted). The IW technology served as a cognitive tool that helped the learners scaffold their own learning early on in the activity. Our results support the conclusion that the technology helped establish shared understanding early on in the activity and consequently human tutoring was not needed as much as it would be without the technology tools. Given that authentic learning activities are socially situated, cognitively structured and affectively charged, they should not be examined in isolation from communicative activities and technology using behavior.

\section{Discussion and Conclusions}

The research described in this paper has provided the reader with examples of concurrent methods used to develop models of the individual learner as well as co-learners engaged in medical problem solving using technology. A mixed method approach was taken that combined qualitative and quantitative data from multiple sources (audio, video and computer trace data) in an effort to provide complimentary types of evidence about medical reasoning about patient cases. Our research goal was to use multiple data sources to create reliable and valid interpretations of the diagnostic reasoning processes by providing the corresponding context in which reasoning took place.

We chose two examples, one that examined multiple means of extracting information from individual experts about their diagnostic reasoning processes, and one that utilized concurrent methods for describing a collaboration within small groups during a simulation of a deteriorating patient. In the first example, the convergence of data sources served several purposes. The first purpose was to build proficiency models of diagnostic reasoning for specific patient cases. Triangulating the data and summarizing it in the form of interactive visual representations resulted in an accurate interpretation of the expert's reasoning processes. We can say this given the fact that experts made very few changes to the visual representations. These externally represented models served as a reflection and self-assessment device for the experts as well as a validation tool for the researcher. 
In the second example we took up the challenge of studying interactive group learning situations in the context of a medical simulation called the deteriorating patient activity. We used mixed methods to describe learning in a complex activity. Research on decision making, communication, scaffolding, and situated learning informed the types of coding of the collaborations with and without technology. The concurrent methods used in this study included: verbal data between students working together in subgroups; student and teacher discourse in the context of the deterioriating patient activity; and computer annotations shared in the IW condition. Together these multiple methods were used to provide evidence of group decision-making, teacher scaffolding and the intersection between student discourse and technology tool use in the context of the deteriorating patient activity. These multiple methods add to our understanding of student-teacher-technology interactions in this complex medical problem solving situation. The concurrent methods helped describe the context and timing of decision making, the content of the reflection and shared knowledge that is constructed through consensus building as peers reflect on the problem list as it is documented and scaffolded by the instructor.

We provided evidence of how one might develop the type of domain-based models of learning and performance suggested by the National Research Council (2001) as a precursor and guide to better forms of assessment. We have started to demonstrate how learning trajectories can be identified as a first step in developing a model of proficiency in these situations. We have also tried to delineate different techniques that can be used to understand both the individual learner's understanding as well as the group when social mediation and consensus building are key requirements in a dynamically changing setting. We looked at the role of technology in both settings from the perspective of instruction, assessment and research. Our goal has been to convey the importance of the interdependence of these tools when establishing more informed interpretations of the phenomenon in question.

Having said this we do realize that there are other mixed method approaches that can inform future work in this area. For example, we could have examined the social construction of knowledge from an activity theory perspective (Engestrom, 1999; Leontiev, 1978, Vygotsky, 1978) that could have provided a different and more detailed look at the complex interactions between persons, tools, and task in terms of the division of labor within the community. Other mechanisms could have been used to document the social structures that exist in the data. For instance, Martinez, Dimitriadis, Rubia, Gomez, and De la Fuente (2003) combined qualitative data (observations, questionnaires, interviews) with quantitative (occurrence of actions or events) and social network analysis (measuring and analyzing relationships and flow among different people who are connected in an activity using graphs to represent the sociometrics of the social network). Other instruments can also serve to document complex activities. For example, CORDTRA (Luckin et al., 2001) provides a chronologically-ordered representation of discourse and tool-related activity. Hmelo (2003) used this tool in her research to interpret the relationship between group discourse and a drawing activity on a whiteboard. In summary, there 
are other methods that can be used to document the co-construction of knowledge building.

Our goal was to demonstrate that diverse indicators of learning and performance can be used to build robust accounts of learning in complex situations. Mixed methods and multiple mediums of assessment can be an asset especially when they converge to provide a valid account of the learning in context.

\section{Acknowledgements}

Send Correspondence to Susanne P. Lajoie, 3700 McTavish St., Faculty of Education, McGill University, Montreal Quebec Canada H3A 1Y2. Funding was provided by the following granting agencies: the Canadian Social Sciences and Humanities Research Council. A special thank you to Jeffrey Wiseman for his inspiration as a medical teacher and researcher. Another special thank you to Marguerite Roy for editorial assistance.

\section{References}

Barrows, H. S. (1986). A taxonomy of problem-based learning methods. Medical Education, 20, 481-486.

Brown, J. S., Collins, A., \& Duguid, P. (1989). Situated cognition and the culture of learning. Educational Researcher, 18(1), 32-42.

Campbell, D. T., \& Fiske, D. W. (1959). Convergent and discriminant validation by the multitraitmultimethod matrix. Psychological Bulletin, 56, 81-105.

Collins, A., Brown, J. S., \& Newman, S. E. (1989). Cognitive apprenticeship: Teaching the crafts of reading, writing, and mathematics. In R. Glaser, \& L. B. Resnick (Eds.), Knowing, learning, and instruction: Essays in honor of Robert Glaser (pp. 453-494). Hillsdale, NJ: Lawrence Erlbaum Associates.

Collins, A., Hawkins, J., \& Frederiksen, J. (1994). Three different views of students: The role of technology in assessing student performance. The Journal for the Learning Sciences, 3(2), 205-217.

Creswell, J. (2003). Research design (2nd ed.). Thousand Oaks, CA: Sage.

Derry, S. J., \& Hmelo-Silver, C. E. (2005). Reconceptualizing teacher education: Supporting case-based instructional problem solving on the World Wide Web. In L. PytlikZillig, M., Bodvarsson, \& R. Bruning (Eds.), Technology based education: Bringing researchers and practitioners together (pp. 21-38). Greenwich, CT: Information Age Publishing.

Engestrom, Y. (1999). Activity theory and individual and social transformation. In Y. Engstrom, R. Miettinen, \& R. Punamaki (Eds.), Perspectives on activity theory (pp. 19-38). NY: Cambridge University Press.

Frederiksen, J. R., \& Collins, A. (1989). A systems approach to educational testing. Educational Researcher, 18(9), 27-32.

Greeno, J. (1989). A perspective on thinking. American Psychologist, 44, 134-141.

Henderson, C., Yerushalmi, E., Heller, K., Heller, P., \& Kuo, V. H. (2003). Multi-Layered Concept Maps for the Analysis of Complex Interview Data. In Proc. physics education research conference, Madisson, WY.

Hmelo-Silver, C. (2003). Analyzing collaborative knowledge construction: Multiple methods for integrated understanding, Computers \& Education, 41, 397-420. 
Johnson, D. W., \& Johnson, R. T. (Eds.) (1999). Learning together and alone: Cooperative, competitive, and individualistic learning (5th ed.). Boston, MA: Allyn \& Bacon.

Khoshafian, S., \& Buckiewicz, W. (1995). Introduction to groupware, workflow, and workgroup computing, John Wiley Publishing, Toronto.

Kolodner, J. L. (1993). Case-based reasoning. San Francisco, CA: Morgan Kaufmann.

Kolodner, J. L., Cox, M. T., \& Gonzalez-Calero, P. (2006). Case-based reasoning-inspired approaches to education. The Knowledge Engineering Review, 20(3), 299-303.

Lajoie, S. P. (2003). Transitions and trajectories for studies of expertise. Educational Researcher, 32(8), 21-25.

Lajoie, S. P. (2007). Developing computer based learning environments based on complex performance models. In W. Spaulding, \& J. Poland (Eds.), Nebraska Symposium on Motivation. Modeling complex systems: Motivation, cognition, and social processes (Vol. 52, pp. 123-144). Lincoln: University of Nebraska Press.

Lajoie, S. P. (2009). Developing professional expertise with a cognitive apprenticeship model: Examples from Avionics and Medicine. In K. A. Ericsson (Ed.). The development of professional performance: Approaches to objective measurement and designed learning environments (pp. 61-83). Cambridge University Press.

Lajoie, S. P., Faremo, S., \& Wiseman, J. (2001). Tutoring strategies for effective instruction in internal medicine. International Journal of Artificial Intelligence and Education, 12(3), 293-309.

Lajoie, S. P., Gauthier, G., \& Richard, S. (2007). Computer tools to support medical problem solving. Paper presented at the European Association for Research on Learning and Instruction (Earli). Budapest, Hungary.

Lajoie, S. P., Lavigne, N., Guerrera, C. P., \& Munsie, S. D. (2001). Constructing knowledge in the context of BioWorld. Instructional Science, 29(2), 155-186.

Lajoie, S. P., \& Lesgold, A. M. (1992). Dynamic assessment of proficiency for solving procedural knowledge tasks. Educational Psychologist, 27(3), 365-384.

Lave, J. (1991). Situated learning in communities of practice. In L. B. Resnick, J. M. Levine, \& S. D. Teasley (Eds.), Perspectives on socially shared cognition (pp. 6382). Washington, DC: American Psychological Association.

Leontiev, A. N. (1978). Activity, consciousness, and personality. Hillsdale: Prentice-Hall.

Lu, J., \& Lajoie, P. S. (2008). Supporting medical decision making with argumentation tools. Contemporary Educational Psychology, 33, 425-442.

Lu, J., Lajoie, S. P., \& Wiseman, J. (submitted). Scaffolding collaboration in simulation based medical emergencies. Instructional Science.

Martinez, A., Dimitriadis, Y., Rubia, B., Gomez, E., \& de la Fuente, P. (2003). Combining qualitative evaluation and social network analysis for the study of classroom social interactions, Computers \& Education, 41, 353-368.

Mislevy, R. J. (2007). Validity by Design. Educational Researcher, 36(8), 463-469.

Mislevy, R. J., Steinberg, L. S., Breyer, F. J., Johnson, L., \& Almond, R. G. (2002). Making sense of data from complex assessments. Applied Measurement in Education, $15,363-378$.

National Research Council. (2001). Implications of the new foundations for assessment design. In J. W. Pellegrino, N. Chudowsky, \& R. Glaser (Eds.), Knowing what students know: The science and design of educational assessment (pp. 176-219). Washington, DC: National Academy Press.

Novak, J. D., \& Cañas, A. J. (2006). The Theory Underlying Concept Maps and How to Construct Them. Technical Report IHMC CmapTools 2006-01: Florida Institute for Human and Machine Cognition. 
O'Donoghue, T., \& Punch K. (2003). Qualitative educational research in action: Doing and reflecting. London, Routledge Falmer.

Pea, R. D. (2004). The social and technological dimensions of scaffolding and related theoretical concepts for learning, education, and human activity. Journal of the Learning Sciences, 13, 423-451.

Pellegrino, J. W., Chudowsky, N., \& Glaser, R. (Eds.) (2001). Knowing what students know: The science and design of educational assessment. Washington, DC: National Academy Press.

Reiser, B. J. (2004). Scaffolding complex learning: The mechanisms of structuring and problematizing student work. Journal of the Learning Sciences, 13(3), 273-304.

Saab, N., van Joolingen, W. R., \& van Hout-Wolters, B. H. (2005). Communication in collaborative discovery learning. British Journal of Educational Psychology 75, 603621.

Schank, R. C. (1998). Inside multi-media case based instruction. Mahwah, NJ: Erlbaum.

Shepard, L. A. (2000). The role of assessment in a learning culture. Educational Researcher, 29(7), 4-14.

van Boxtel, C. (2000). Collaborative concept learning: Collaborative learning tasks, student interaction, and the learning of physics concepts. Unpublished Doctoral thesis, Utrecht University, Utrecht, Netherlands.

Vygotsky, L. S. (1978). Mind in Society. Cambridge, MA: Harvard University Press.

Wiseman, J., \& Snell, L. (2008). The deteriorating patient: A realistic but "low-tech" simulation of emergency decision-making. The Clinical Teacher, 5, 93-97.

Wood, D., Bruner, J. S., \& Ross, G. (1976). The role of tutoring in problem solving. Journal of Child Psychology \& Psychiatry 85 Allied Disciplines, 17(2), 89-100.

Woods, D. K., \& Fassnacht, C. (2007). Transana (Version 2.20). Madison, WI: The Board of Regents of the University of Wisconsin System. 